\title{
Effect of Anisotropy on the Critical Behaviour of Three- Dimensional Heisenberg Ferromagnets
}

\author{
R. Shanker and R. A. Singh \\ Department of Physics, University of Gorakhpur, Gorakhpur 273001, India
}

(Z. Naturforsch. 31 a, 34-40 [1976] ; received September 15, 1975)

\begin{abstract}
The anisotropic nearest-neighbour Heisenberg model for the simple cubic lattice has been investigated by interpolating the anisotropy between the Ising and isotropic Heisenberg limits via general spin high-temperature series expansions of the zero-field suspectibility. This is done by estimating the critical temperature $\left(T \mathrm{c}^{(3)}\right)$ and the susceptibility exponent $\gamma$ from the analysis of the series by the Ratio and Pade approximants methods. It is noted that $T_{\mathrm{c}}{ }^{(3)}$ varies with anisotropy while $\gamma$ is almost the same for the anisotropic system, and a jump in it occurs for the isotropic case in agreement with the universality hypothesis. The effect of anisotropy on the susceptibility is also shown. Further, it is seen that estimates of $\gamma$ for the two extreme limits agree well with those of previous theoretical as well as experimental investigations. In addition, critical temperatures have been summarised in a relation, and expressions for the magnetisation have been derived.
\end{abstract}

\section{Introduction}

Critical indices (exponents) of the various physical quantities describe the nature and strength of their singularities and are, therefore, key parameters in characterising critical point phenomena. Because of this fact many investigations concerning the critical phenomena, both experimental and theoretical, have centred on the determination of the exponents. The determination of the exponents has also been motivated to confirm or to disprove the scaling and universality hypotheses ${ }^{1,2}$. The Ising and Heisenberg models have, in particular, been chosen for such investigations because of their relevance to real ferro- and antiferromagnets, and also because they can be viewed as mathematically simple and physically very general forms of a classical and quantum mechanical many body problem. The series expansion method has proven to be the most fruitful technique to determine the various critical exponents for both the models on different lattices.

In both the models the occurence of a phase transition is closely connected with the existence of spontaneous magnetisation. In the Ising model the onedimensional (1-d) lattice does not exhibit a phase transition ${ }^{3}$ but the two- and three-dimensional (2-d and 3-d) lattices have a phase transition ${ }^{4-6}$. On the other hand, in the Heisenberg model 1-d and 2-d lattices have no phase transition $^{7}$ while 3 -d lattices have a phase transition ${ }^{8}$. Because of this fact much attention has been paid to the critical behaviour of 3-d Ising and Heisenberg models. It has been found ${ }^{9-11}$ that the critical exponents are the same for all 3-d lattices in agreement with the universality belief.
Further, considerable efforts have been made in the last ten years to see the effect of change in the symmetry of the order parameter (change from an Ising to an isotropic Heisenberg system) on the critical behaviour. Dalton and Wood ${ }^{12}$ have investigated the anisotropic nearest-neighour (nn) Heisenberg model by analysing high-temperature series (HTS) of five terms for the zero field susceptibility for spin $1 / 2$ by the Padé approximant method. Similar investigations have been presented by Jou and Chen ${ }^{13}$ for spin $1 / 2$ using seven terms series, and by Wood and Fox ${ }^{14}$ for $S=1 / 2,1$ and $3 / 2$ using six terms series. These workers have interpolated, with anisotropy, between the Ising and Heisenberg limits and concluded that the susceptibility exponent $\gamma$ for 3 -d cubic (bcc and fcc) lattices remains Ising like in the presence of anisotropy and increases discontinuously in the isotropic Heisenberg limit. This is in agreement with the conclusion drawn by Jasnow and Wortis ${ }^{15}$ by analysing eight terms series of different physical quantities for the $S=\infty$ anisotropic nn Heisenberg model for 3-d fcc lattice. In an investigation for the simple cubic (sc) lattice by Obokata et al. ${ }^{16}$ for spin $1 / 2$ no clear conclusion has been drawn. Hence we have investigated the anisotropic nn Heisenberg model for sc lattice by analysing general spin HTS ${ }^{17}$ of six terms of the zero-field susceptibility. This is done by estimating the critical parameters by both the Ratio- and Pade-methods for comparison. These are discussed in the light of existing investigations. In addition transition temperatures are summarised in a relation, and expressions for the magnetisation are derived. The effect of anisotropy on the susceptibility is also shown.

Reprint requests to Dr. R. A. Singh, Department of Physics, University of Gorakhpur, Gorakhpur 273001, India. 


\section{Theoretical Details and Results}

\subsection{High Temperature Zero-Field Susceptibility Series}

The anisotropic Heisenberg spin Hamiltonian ${ }^{17}$ for the present investigation may be given in the form

$$
\begin{gathered}
\mathcal{H}=-2 J \sum_{i j}\left\{S_{i z} S_{j z}+2 \eta\left(S_{i x} S_{j x}+S_{i y} S_{j y}\right)\right\} \\
-\mu H \sum_{i=1}^{N} S_{i z},
\end{gathered}
$$

where $\eta$ is the anisotropy parameter and the other symbols have their usual meaning. The Ising and isotropic Heisenberg models correspond to $\eta=0$ and $\eta=0.5$ respectively. The expansion of the zero field susceptibility $\chi_{0}$, corresponding to (1), can be written in the form ${ }^{17}$

$$
\frac{k_{\mathrm{B}} T \chi_{0}}{\mu^{2}}=\frac{X}{3}\left\{1+\sum_{n=1}^{\infty} a_{n}(\eta, X) K^{n}\right\},
$$

where $X=S(S+1), K=J X / 2 k_{\mathrm{B}} T$ and the other symbols have their usual meaning. The coefficients $a_{n}$ (up to $n=6$ ) have been calculated from the general formalism of Wood and Dalton ${ }^{17}$ for the sc lattice for different values of $S$ (i.e. for $S=1 / 2$, 1 , $3 / 2,2,3$ and $\infty)$ in the range $0 \leqq \eta \leqq 0.5$. These are presented in Table 1.

\subsection{Estimation of the Critical Temperature and Exponent}

(a) Pade Approximant (PA) method: This is a very powerful method ${ }^{18}$ to estimate the

\begin{tabular}{|c|c|c|c|c|c|c|c|}
\hline$S$ & $\eta$ & 0.0 & 0.1 & 0.2 & 0.3 & 0.4 & 0.5 \\
\hline \multirow{6}{*}{1} & $a_{1}$ & 8.00000 & 8.00000 & 8.00000 & 8.00000 & 8.00000 & 8.00000 \\
\hline & $a_{2}$ & 53.33333 & 52.90667 & 51.62667 & 49.49333 & 46.50667 & 44.23111 \\
\hline & $a_{3}$ & 350.81481 & 345.12592 & 328.05925 & 299.61481 & 259.79259 & 208.59259 \\
\hline & $a_{4}$ & 2231.30864 & 2180.37665 & 2029.27976 & 1783.11522 & 1450.41383 & 1042.96296 \\
\hline & $a_{5}$ & 14162.38354 & 13744.89495 & 12523.17445 & 10589.45790 & 8097.47174 & 5262.43292 \\
\hline & $a_{6}$ & 88512.55583 & 84793.24530 & 76064.68280 & 61096.83753 & 43980.54564 & 24731.82401 \\
\hline \multirow{6}{*}{$1 / 2$} & $a_{1}$ & 8.00000 & 8.00000 & 8.00000 & 8.00000 & 8.00000 & 8.00000 \\
\hline & $a_{2}$ & 56.00000 & 55.73333 & 54.93333 & 53.60000 & 51.73333 & 49.33333 \\
\hline & $a_{3}$ & 389.33333 & 385.42222 & 373.68889 & 354.13333 & 326.75556 & 291.55556 \\
\hline & $a_{4}$ & 2623.11111 & 2584.66299 & 2469.31010 & 2277.02684 & 2007.77055 & 1661.48148 \\
\hline & $a_{5}$ & 17641.36294 & 17298.88838 & 16277.68070 & 14596.38803 & 12277.49080 & 9390.30125 \\
\hline & $a_{6}$ & 116995.67411 & 114186.29407 & 105945.24529 & 92504.08535 & 74346.00981 & 52053.74814 \\
\hline \multirow{6}{*}{$3 / 2$} & $a_{1}$ & 8.00000 & 8.00000 & 8.00000 & 8.00000 & 8.00000 & 8.00000 \\
\hline & $a_{2}$ & 56.74667 & 56.52480 & 55.85920 & 54.74986 & 53.19680 & 51.20000 \\
\hline & $a_{3}$ & 400.33090 & 396.99403 & 386.98340 & 370.29903 & 346.94091 & 316.90904 \\
\hline & $a_{4}$ & 2742.08490 & 2708.59435 & 2607.81163 & 2437.92604 & 2204.52959 & 1889.26736 \\
\hline & $a_{5}$ & 18752.29792 & 18448.27136 & 17536.33088 & 16016.90118 & 13890.68928 & 11158.65601 \\
\hline & $a_{6}$ & 126541.07222 & 124012.81650 & 116474.58410 & 104007.46590 & 86733.39001 & 64749.83489 \\
\hline \multirow{6}{*}{2} & $a_{1}$ & 8.00000 & 8.00000 & 8.00000 & 8.00000 & 8.00000 & 8.00000 \\
\hline & $a_{2}$ & 57.06667 & 56.86400 & 56.25600 & 55.24267 & 53.82400 & 52.00000 \\
\hline & $a_{3}$ & 405.07259 & 401.99206 & 392.75046 & 377.34779 & 355.78406 & 328.05926 \\
\hline & $a_{4}$ & 2794.33878 & 2763.18028 & 2669.30775 & 2511.41860 & 2287.49912 & 1994.61729 \\
\hline & $a_{5}$ & 19247.73696 & 18962.88896 & 18106.12455 & 16670.77890 & 14645.74438 & 12015.47059 \\
\hline & $a_{6}$ & 130867.99937 & 128479.44910 & 121326.51541 & 109414.61670 & 92724.14167 & 71152.75141 \\
\hline \multirow{6}{*}{3} & $a_{1}$ & 8.00000 & 8.00000 & 8.00000 & 8.00000 & 8.00000 & 8.00000 \\
\hline & $a_{2}$ & 57.33333 & 57.14667 & 56.58667 & 55.65333 & 54.34667 & 52.66667 \\
\hline & $\overline{a_{3}}$ & 409.03704 & 406.17481 & 397.58839 & 383.27703 & 363.24148 & 337.48148 \\
\hline & $a_{4}$ & 2838.55767 & 2809.34912 & 2721.50464 & 2573.46424 & 2362.77856 & 2086.01848 \\
\hline & $a_{5}$ & 19669.62438 & 19402.05952 & 18595.31187 & 17237.20755 & 15307.46240 & 12777.67814 \\
\hline & $a_{6}$ & 134584.91551 & 132325.01801 & 125534.16008 & 114155.91955 & 98058.87120 & 76983.14200 \\
\hline \multirow{6}{*}{$\infty$} & $a_{1}$ & 8.00000 & 8.00000 & 8.00000 & 8.00000 & 8.00000 & 8.00000 \\
\hline & $a_{2}$ & 57.60000 & 57.42933 & 56.91733 & 56.06400 & 54.86933 & 53.33330 \\
\hline & $\overline{a_{3}}$ & 413.01333 & 410.37369 & 402.45476 & 389.25653 & 370.77902 & 347.02222 \\
\hline & $a_{4}$ & 2883.12889 & 2856.14019 & 2774.62070 & 2636.91014 & 2440.24145 & 2180.74074 \\
\hline & $a_{5}$ & 20099.81194 & 19850.75110 & 19097.81133 & 17823.72150 & 15999.69325 & 13585.42786 \\
\hline & $a_{6}$ & 138405.06470 & 136286.53691 & 129896.56924 & 119119.74981 & 103723.14808 & 83296.21748 \\
\hline
\end{tabular}
critical parameters from the truncated series. The

Table 1. The coefficients $a_{n}$ of the expansion (2) for different values of $S$ and $\eta$. 
susceptibility series of the present investigation, near the critical point, may be defined by the relationship 9,19

$$
\chi=A\left(1-K / K_{\mathrm{c}}\right)^{-\gamma},
$$

where $\quad \chi=3 k_{\mathrm{B}} T \chi_{0} / \mu^{2} X, \quad K_{\mathrm{c}}=J X / 2 k_{\mathrm{B}} T_{\mathrm{c}}^{(3)}$ $\left(T_{\mathrm{c}}^{(3)}\right.$ stands for the temperature at which the susceptibility diverges for 3-d systems), $A$ is the amplitude and $\gamma$ the critical exponent. Estimates of $K_{\mathrm{c}}$ are obtained from the appropriate poles (zeros of the denominator) of the PA's to $\mathrm{d}(\ln \chi) / \mathrm{d} K$. The Pade analysis of $\mathrm{d}(\ln \chi) / \mathrm{d} K$ for $S=3$ with $0 \leqq \eta \leqq 0.5$ is presented in Table 2 . This table shows that estimates of $K_{\mathrm{c}}$ from higher order approximants are in good agreement with each other. Antiferromagnetic singularities $K_{1}$ have also been estimated with a view to estimate $K_{\mathrm{c}}$ from the PA's to $\left(K-K_{1}\right) \mathrm{d}(\ln \chi) / \mathrm{d} K$. This procedure ${ }^{20,21}$ removes the antiferromagnetic singularities and improves the consistency in $K_{\mathrm{c}}$. But the $K_{1}$ were found irregular, which prevented us from doing such a refining. The estimates of $\gamma$ are made from the values of the higher order PA's to $\left(K_{\mathrm{c}}-K\right) \mathrm{d}(\ln \chi) / \mathrm{d} K$ at $K=K_{\mathrm{c}}$. These are presented in Table 3 for $S=3$ in the range $0 \leqq \eta \leqq 0.5$. It has been seen that a change of 0.0005 in the value of $K_{\mathrm{c}}$ leads to a change of about 0.01 in the value of $\gamma$. The estimated $K_{\mathrm{c}}$ and $\gamma$ from the higher order PA's to $\mathrm{d}(\ln \chi) / \mathrm{d} K$ and $\left(K_{c}-K\right) \mathrm{d}(\ln \chi) / \mathrm{d} K$ respectively for different values of $S$ and $\eta$ are presented in Table 4 . The uncertainty limits, which are somewhat subjective, are also given in Table 4 .

(b) Ratio Method: The estimates of $K_{c}^{-1}$ are made by plotting square roots of the ratios of alternate coefficients as a function of $1 / n$ and extrapolating for $n=\infty$. This procedure ${ }^{18}$ removes the antiferromagnetic singularities (causing oscillations in the ratios of the successive coefficients) and gives reliable estimates for all $\eta(0 \leqq \eta \leqq 0.5)$. An example for $S=3$ is presented in Figure 1. The critical temperatures, thus estimated, are presented in Table 5. The estimates of $\gamma$ are made from the sequences of the $\gamma_{n}=\left(r_{n} K_{c_{1}}^{-1}-1\right) n+1$ where $r_{n}=$ $a_{n} / a_{n-1}$. These are also listed in Table 5 . The inverse of $K_{\mathrm{c}}$ as a function of $\eta$ for different spins is shown in Figure 2.

Table 2. Estimates of $K_{\mathrm{c}}$ for $S=3$ with different values of $\eta$ from the higher order Pade approximants to d(ln $\left.\chi\right) / \mathrm{d} K$.

* Denotes the complex pole.

\begin{tabular}{|c|c|c|c|c|c|c|c|c|c|c|c|}
\hline$D$ & $N 1$ & $\begin{array}{c}\eta=0 \\
2\end{array}$ & 3 & $D$ & $N 1$ & $\begin{array}{c}\eta=0.1 \\
2\end{array}$ & 3 & $D$ & $N 1$ & $\begin{array}{c}\eta=0.2 \\
2\end{array}$ & 3 \\
\hline \multirow{5}{*}{$\begin{array}{l}2 \\
3 \\
4 \\
\mathrm{E}\end{array}$} & 0.1489 & 0.1503 & \multirow[t]{3}{*}{0.1508} & 2 & 0.1496 & 0.1507 & \multirow[t]{3}{*}{0.1512} & 2 & 0.1515 & 0.1521 & \multirow[t]{5}{*}{0.1527} \\
\hline & 0.1504 & \multirow[t]{2}{*}{0.1512} & & 3 & 0.1508 & \multirow[t]{2}{*}{0.1518} & & 3 & 0.1522 & \multirow[t]{2}{*}{0.1540} & \\
\hline & 0.1509 & & & 4 & 0.1513 & & & 4 & 0.1527 & & \\
\hline & Estimates & \multicolumn{2}{|c|}{$0.1508 \pm 0.0004$} & & & \multicolumn{2}{|c|}{$0.1512 \pm 0.0005$} & & \multicolumn{2}{|c|}{$0.1525 \pm 0.0005$} & \\
\hline & & \multicolumn{2}{|l|}{$\eta=0.3$} & \multicolumn{4}{|c|}{$\eta=0.4$} & & \multicolumn{2}{|r|}{$\eta=0.5$} & \\
\hline 2 & 0.1554 & 0.1552 & 0.1553 & 2 & 0.1620 & 0.1613 & 0.1614 & 2 & 0.1740 & 0.1744 & 0.1755 \\
\hline 3 & 0.1560 & * & & 3 & * & 0.1614 & & 3 & 0.1743 & 0.1743 & \\
\hline \multirow{2}{*}{\multicolumn{2}{|c|}{ Estimates }} & \multirow{2}{*}{\multicolumn{2}{|c|}{$0.1654 \pm 0.0005$}} & 4 & 0.1614 & & & 4 & \multirow{2}{*}{\multicolumn{2}{|c|}{$0.1747 \pm 0.0008$}} & \\
\hline & & & & & & \multicolumn{2}{|c|}{$0.1614 \pm 0.0005$} & & & & \\
\hline
\end{tabular}

Table 3. Estimates of $\gamma$ for $S=3$ with different values of $\eta$ from higher order Pade approximants to $\left(K_{\mathrm{c}}-K\right) \mathrm{d}(\ln \eta) / \mathrm{d} K$ at $K=K_{\mathrm{c}}$ (the values of $K_{\mathrm{c}}$ are taken from Table 2).

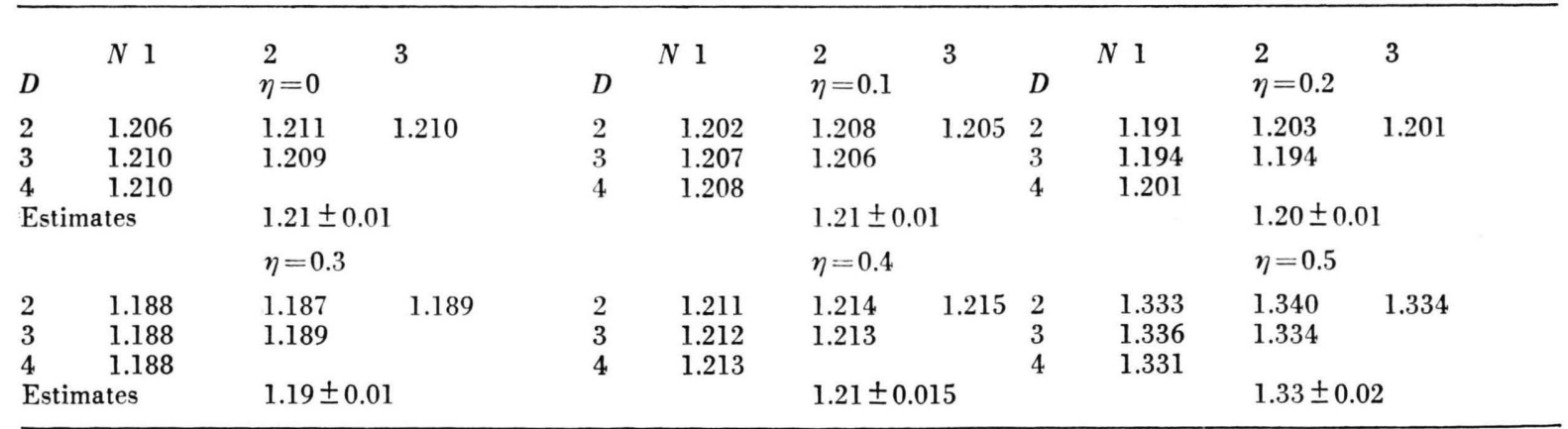


Table 4. Estimates of $K_{\mathrm{c}}$ (upper line) and $\gamma$ (lower line) for various values of $S$ and $\eta$ from the higher order Pade approximants.

\begin{tabular}{|c|c|c|c|c|c|c|c|}
\hline$S \eta$ & 0 & 0.1 & 0.2 & 0.3 & 0.4 & 0.5 & \\
\hline$\frac{1}{2}$ & $0.1661 \pm 0.0007$ & $0.167 \pm 0.001$ & $0.170 \pm 0.002$ & $0.178 \pm 0.003$ & $0.185 \pm 0.005$ & 0.21 & \pm 0.02 \\
\hline & $0.1561 \pm 0.0005$ & $0.1569 \pm 0.0005$ & $0.1588 \pm 0.0007$ & $0.1627 \pm 0.0007$ & $0.1 \overline{7} \pm 0.001$ & $0 . \overline{192}$ & \pm 0.001 \\
\hline $\mathrm{l}$ & $1.24 \pm 0.01$ & $1.24 \pm 0.01$ & $1.23 \pm 0.01$ & $1.22 \pm 0.02$ & $1.24 \pm 0.02$ & 1.38 & \pm 0.03 \\
\hline & $0.1529 \pm 0.0005$ & $0.1538 \pm 0.0005$ & $0.1550 \pm 0.0005$ & $0.1590 \pm 0.0006$ & $0.1661 \pm 0.0006$ & 0.182 & \pm 0.001 \\
\hline$\frac{\frac{3}{2}}{2}$ & $1.23 \pm 0.01$ & $1.23 \pm 0.01$ & $1.22 \pm 0.01$ & $1.21 \pm 0.015$ & $1.23 \pm 0.015$ & 1.36 & \pm 0.02 \\
\hline & $0.1517 \pm 0.0005$ & $0.1524 \pm 0.0005$ & $0.1539 \pm 0.0005$ & $0.1571 \pm 0.0005$ & $0.1634 \pm 0.0006$ & 0.178 & \pm 0.001 \\
\hline 2 & $1.22 \pm 0.01$ & $1.22 \pm 0.01$ & $1.21 \pm 0.01$ & $1.20 \pm 0.015$ & $1.22 \pm 0.015$ & 1.34 & \pm 0.02 \\
\hline 3 & $0.1508 \pm 0.0004$ & $0.1512 \pm 0.0005$ & $0.1525 \pm 0.0005$ & $0.1554 \pm 0.0004$ & $0.1614 \pm 0.0005$ & 0.1747 & \pm 0.0008 \\
\hline 3 & $1.21 \pm 0.01$ & $1.21 \pm 0.01$ & $1.20 \pm 0.01$ & $1.19 \pm 0.01$ & $1.21 \pm 0.015$ & 1.33 & \pm 0.02 \\
\hline & $0.1498 \pm 0.0004$ & $0.1501 \pm 0.0005$ & $0.1511 \pm 0.0005$ & $0.1541 \pm 0.0005$ & $0.1595 \pm 0.0005$ & 0.1725 & \pm 0.0008 \\
\hline$\infty$ & $1.20 \pm 0.01$ & $1.20 \pm 0.01$ & $1.20 \pm 0.01$ & $1.19 \pm 0.01$ & $1.21 \pm 0.015$ & 1.33 & \pm 0.02 \\
\hline
\end{tabular}

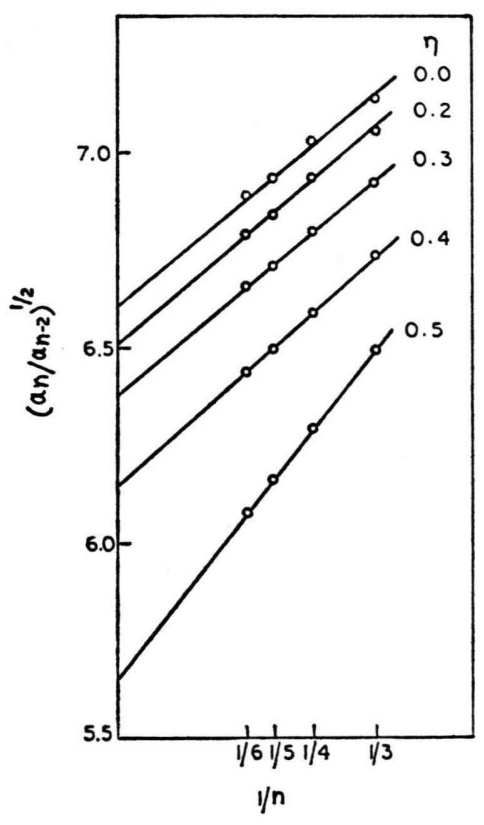

Fig. 1. The ratios $\left(a_{n} / a_{n-2}\right)^{1 / 2}$ of the susceptibility series plotted against $1 / n$ for $S=3$ with different values of $\eta$.

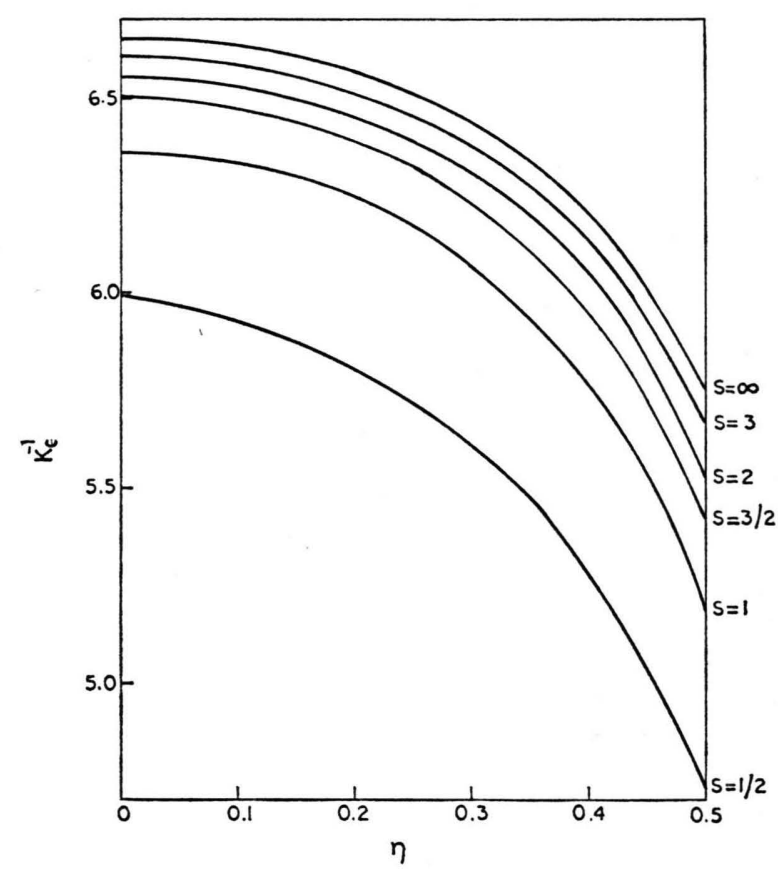

Fig. 2. Variation of the critical temperature with the anisotropy parameter $\eta$ for different values of spin.

Table 5. Estimates of $\mathrm{K}_{\mathrm{c}}^{-1}$ (upper line) and $\gamma$ (lower line) for various values of $S$ and $\eta$ from the ratio method.

\begin{tabular}{|c|c|c|c|c|c|c|}
\hline$S \eta$ & 0.0 & 0.1 & 0.2 & 0.3 & 0.4 & 0.5 \\
\hline \multirow{2}{*}{$\frac{1}{2}$} & 5.99 & 5.94 & 5.82 & 5.57 & 5.33 & 4.74 \\
\hline & $1.25 \pm 0.04$ & $1.25 \pm 0.05$ & $1.27 \pm 0.05$ & $1.28 \pm 0.05$ & - & - \\
\hline \multirow{2}{*}{1 . } & 6.36 & 6.33 & 6.25 & 6.08 & 5.78 & 5.19 \\
\hline & $1.25 \pm 0.02$ & $1.25 \pm 0.02$ & $1.25 \pm 0.02$ & $1.25 \pm 0.02$ & $1.27 \pm 0.02$ & $1.42 \pm 0.03$ \\
\hline \multirow{2}{*}{$\frac{3}{2}$} & 6.50 & 6.46 & 6.40 & 6.24 & 5.96 & 5.43 \\
\hline & $1.24 \pm 0.02$ & $1.24 \pm 0.02$ & $1.24 \pm 0.02$ & $1.24 \pm 0.02$ & $1.27 \pm 0.02$ & $1.41 \pm 0.02$ \\
\hline \multirow{2}{*}{2} & 6.55 & 6.52 & 6.45 & 6.32 & 6.07 & 5.55 \\
\hline & $1.23 \pm 0.02$ & $1.23 \pm 0.02$ & $1.23 \pm 0.02$ & $1.23 \pm 0.02$ & $1.26 \pm 0.02$ & $1.40 \pm 0.02$ \\
\hline \multirow{2}{*}{3} & 6.61 & 6.59 & 6.51 & 6.38 & 6.15 & 5.66 \\
\hline & $1.22 \pm 0.02$ & $1.22 \pm 0.02$ & $1.22 \pm 0.02$ & $1.22 \pm 0.02$ & $1.25 \pm 0.02$ & $1.39 \pm 0.02$ \\
\hline \multirow{2}{*}{$\infty$} & 6.65 & 6.63 & 6.56 & 6.45 & 6.22 & 5.76 \\
\hline & $1.22 \pm 0.02$ & $1.22 \pm 0.02$ & $1.22 \pm 0.02$ & $1.22 \pm 0.02$ & $1.25 \pm 0.02$ & $1.38 \pm 0.02$ \\
\hline
\end{tabular}


Next, $k_{\mathrm{B}} T^{(\mathbf{3})} / \mathrm{J}$ has been plotted as a function of $X$ for different values of $\eta(0 \leqq \eta \leqq 0.5)$ in Figure 3. The remarkable feature of Fig. 3 is that

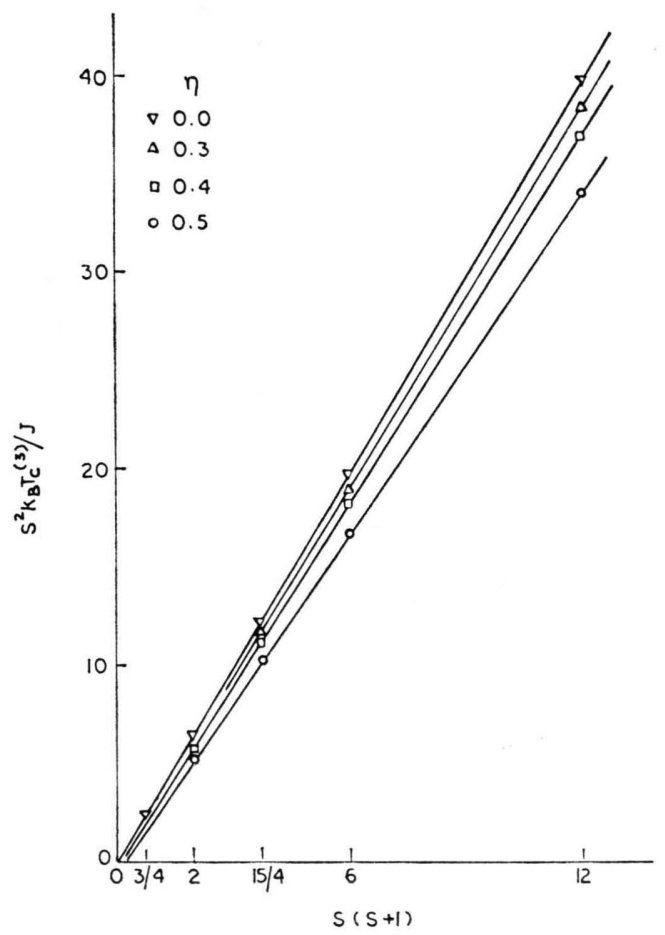

Fig. 3. Variation of $k_{\mathrm{B}} T_{\mathrm{c}}{ }^{(3)} / J$ with $S(S+1)$.

the critical points lie on straight lines for any $\eta(0 \leqq \eta \leqq 0.5)$. From this property the critical temperatures are summarised in the equation

$$
\begin{aligned}
k_{\mathrm{B}} T_{\mathrm{c}}^{(3)} / J \cong & \left(3.33-3.6 \eta^{3}\right) \\
\cdot & {\left[S(S+1)-\left(0.075+0.85 \eta^{3}\right)\right], }
\end{aligned}
$$

\subsection{Estimation of $\chi$}

The presence of interfering singularities prevented us from getting a reliable estimate of $\gamma$ as a function of temperature by the PA method. Therefore the estimation of $\chi$ as a function of temperature (i.e. $K^{-1}$ ) has been made by plotting $\chi$ as a function of $1 / n$ and extrapolating for $n=\infty$. Results thus obtained for $S=3$ with $0 \leqq \eta \leqq 0.5$ are shown in Figure 4. Similar plots can be obtained for other spins.

\section{4- Spin-Wave Results for Magnetisation}

It is well known that the results of the spin-wave theory are excellent at low temperatures for practical purposes. With this view expressions for the magnetisation, valid for any spin for the sc lattice

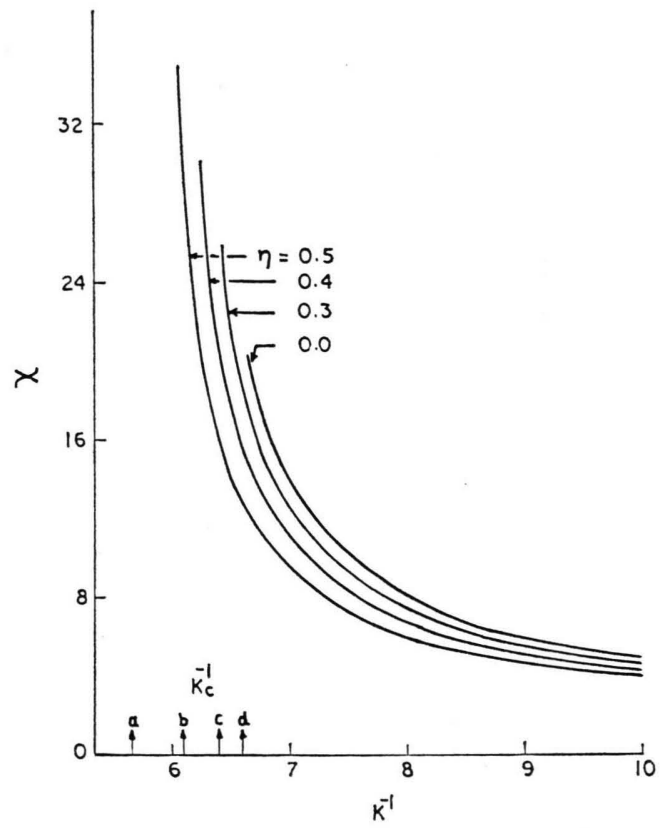

Fig. 4. The zero-field susceptibility as a function of the temperature for $S=3$ with $\eta$ as a parameter. The arrows $a, b$, $c$, and $d$ indicate the critical temperatures $K_{\mathrm{c}}{ }^{-1}$ (located from the ratio method corresponding to $\eta=0.5,0.4,0.3$ and 0.0 respectively.

defined by the Hamiltonian (1), are derived by using the ideas of the spin-wave theory ${ }^{22,23}$. For sufficiently low temperatures, the physical assumptions of the theory are: (i) there exists an ordered ground state characterised by a magnetisation in the $z$ direction and (ii) deviations from the ordered ground state are small. Hence $S_{x} \ll S, \quad S_{y} \ll S$ and $S-S_{z} \ll S$ where $S_{x}, S_{y}$ and $S_{z}$ are respectively the components of the classical spin $S$ in the $x, y$ and $z$ directions. by 22

The magnetisation at low temperature is given

$$
\bar{m}=S-\frac{V_{0}}{(2 \pi)^{d}} \int_{B Z} n_{k} \mathrm{~d} \boldsymbol{k}
$$

where the integral is over the Brillouin zone, $V_{\mathbf{0}}$ is the volume of a unit cell, $d$ is the dimensionality and $n_{k}$ is the wave occupation number.

Next, in order to get expressions for the magnetisation a set of dynamical equations for the spins were obtained using the ideas of the spin-wave theory and Hamiltonian (1). The set of linear equations, thus obtained, was reduced to an approximate set of linear equations by taking $S_{j z}=S$ and neglecting terms of the order $S_{i x} S_{j y}$ according to the assumptions of the theory. Further, the normal mode 
frequencies (for each wave vector $\boldsymbol{k}$ ) obtained by Fourier transformation of the reduced equations for simple cubic lattice, are given by

$$
\begin{aligned}
& \omega_{\boldsymbol{k}}=h+12 J S \\
& \cdot\left[1-\frac{2 \eta}{3}\left(\cos k_{x} a+\cos k_{y} a+\cos k_{z} a\right)\right],
\end{aligned}
$$

where $h=\mu H$. In the long wavelength limit Eq. (6) reduces to

$$
\omega_{k}=h+12 J S(1-2 \eta)+4 J S \eta k^{2} a^{2} .
$$

Substituting the low temperature approximation to the wave occupation number $\left(n_{k} \cong k_{\mathrm{B}} T / \omega_{\boldsymbol{k}}=\right.$ $\left.1 / \omega_{\boldsymbol{k}} \beta\right)$ and the long wave length approximation to $\omega_{\boldsymbol{k}}$ in (5), we get

$$
\begin{aligned}
\bar{m}=S-\frac{a^{3}}{2 \beta \pi^{2}} \int_{k=0}^{k_{m}} & \frac{k^{2} \mathrm{~d} k}{h+12 J S(1-2 \eta)+4 J S \eta k^{2} a^{2}} .
\end{aligned}
$$

Since at low temperatures the shape of the Brillouin zone is not important, the upper limit $k_{m}$ may be defined by $k_{m}=(2 \pi / a)(3 / 4 \pi)^{1 / s}$. Hence (8) reduces to

$$
\begin{aligned}
\bar{m}=S & -\frac{1}{4 \pi \eta J S \beta}\left(\frac{3}{4 \pi}\right)^{\frac{1}{3}} \\
& +\frac{1}{16 \pi^{2} \eta J S \beta} \sqrt{\frac{h+12 J S(1-2 \eta)}{J S \eta}}(6) \\
& \cdot \tan ^{-1}(3)^{1 / 3}(4 \pi)^{2 / 3} \sqrt{\frac{J S \eta}{h+12 J S(1-2 \eta)}}
\end{aligned}
$$

This expression is valid for all $\eta$ ecxept for $\eta=0$. The $\bar{m}$ for two extreme cases are given below.

Case (i): The expression for magnetisation for $\eta=1 / 2$ is given by

$$
\bar{m}=S-\frac{1}{2 \pi J S \beta}\left(\frac{3}{4 \pi}\right)^{\frac{1}{3}} .
$$

Case (ii): The expression for magnetisation for $\eta=0$ is given by

$$
\bar{m}=S-\frac{1}{\beta(h+12 J S)} .
$$

\section{Discussion and Conclusions}

(i) Figure 2 sohws that $K_{\mathrm{c}}^{-1}$ (i.e. $T_{\mathbf{k}}^{(3)}$ ) is finite and spin dependent for all values of $\eta(0 \leqq \eta \leqq 0.5)$. It decreases as $\eta$ increases slowly near the Ising limit and rapidly near the isotropic limit for each spin. The curves are qualitatively similar to the curve for $S=\infty$ of the anisotropic nn Heisenberg model for 3 -d fec lattice ${ }^{15}$. The cause of this type of variation of the critical temperature with $\eta$ is clear from the Hamiltonian (1). It can be seen from (1) that if $\eta<0.5$, the relative probability of the spin to become parallel to the $z$ axis is larger than to be in the $x y$ plane. Therefore the critical temperature is higher for smaller $\eta$.

(ii) The behaviour of the exponent $\gamma$, as $\eta$ varies from the Ising limit towards the isotropic Heisenberg limit, is of special interest. Table 4 shows that $\gamma$ for $S>1 / 2$, is Ising like in presence of anisotropy and a jump in it occurs for the isotropic case. A similar trend of $\gamma$ is clear from Table 5 also. The minor anomaly in the ratio estimates of $\gamma$ near the isotropic limit is due to the presence of isotropic behaviour and the short series effects. Thus the behaviour of $\gamma(\eta)$ for $S>1 / 2$, is in agreement with the universality belief in accordance with the previous investigations on the cubic lattices ${ }^{12-15 a}$. It is remarkable to note that estimates of $\gamma$ from the Pade method are lower than those from the ratio method. This is because of the fact that estimates of $K_{\mathrm{c}}$ by the Pade method are lower than those from the ratio method by upto 0.3 to $2 \%$. However, estimates of $\gamma$ by the ratio method for the Ising and isotropic Heisenberg limits are in good agreement with those obtained from the Pade and ratio methods using longer series ${ }^{24-26}$. Hence, critical parameters from the ratio method seem more reliable for all $\eta(0 \leqq \eta$ $\leqq 0.5)$ than those obtained from the Pade method. Equation (4) can be used to locate critical temperatures for any set of $S\left(\frac{1}{2} \leqq S \leqq \infty\right)$ and $\eta(0 \leqq \eta$ $\leqq 0.5)$. Although Fig. 2 can be used for the same purpose; its use is limited to those spins which are given in it.

The mean of the experimental values ${ }^{27}$ of $\gamma$ for the 3-d Heisenberg ferromagnets is known to be 1.35 \pm 0.04 . This is somewhat lower than the present ratio estimate. This difference is due to presence of anisotropy in the compounds. The experimental value $^{27}$ of $\gamma$ (i.e. $1.24 \pm 0.03$ ) for 3 -d Ising compounds agrees also well with the present estimate.

(iii) Figure 4 shows how the susceptibility $\chi$ depends upon anisotropy. It is clear that $\chi$ is very sensitive to large $\eta$ (i.e. small anisotropy) at low temperature and less sensitive to it for $T \gg T_{\mathbf{c}}^{(3)}$.

(iv) Finally, we wish to mention that expressions, (9), (10) and (11) can be used for practical pur- 
poses at low temperatures by making use of the relation $\alpha=H_{\mathrm{A}} / H_{\mathrm{E}}=1-2 \eta$ (where $\alpha$ is the anisotropy which can be determined experimentally, $H_{\mathrm{A}}$ is the anisotropy field and $H_{\mathrm{E}}$ the exchange field).

1 R. B. Griffiths, Phys. Rev. Lett. 24, 1479 [1970].

2 A. Hankey and H. E. Stanley, Phys. Rev. B 6, 3515 [1972].

3 M. E. Baur and L. H. Nosanow, J. Chem. Phys. 37, 153 [1962].

4 C. N. Yang, Phys. Rev. 85, 808 [1952].

5 R. Peierls, Proc. Cambridge Phil. Soc. 32, 477 [1936].

6 R. B. Griffiths, J. Math. Phys. 8, 478 [1967].

7 N. D. Mermin and H. Wagner, Phys. Rev. Lett. 17, 1133 [1966].

8 T. Oguchi, J. Phys. Soc. Japan 30, 988 [1971].

9 M. E. Fisher, Rep. Prog. Phys. 30, 615 [1967].

10 C. Domb, Adv. Physics 19, 339 [1970].

11 R. G. Bowers and M. E. Woolf, Phys. Rev. 177, 917 [1969].

12 N. W. Dalton and D. W. Wood, Proc. Phys. Soc. 90, 459 [1967].

13 D. C. Jou and H. H. Chen, J. Phys. C 6, 2713 [1973].

14 D. W. Wood and P. F. Fox (to be published).

15 D. Jasnow and M. Wortis, Phys. Rev. 176, 739 [1968].

${ }^{15 a}$ P. Pfeuty, D. Jasnow, and M. E. Fisher, Phys. Rev. B 10, 2088 [1974].

16 T. Obokata, I. Ono, and T. Oguchi, J. Phys. Soc. Japan 23, 516 [1967].

\section{Acknowledgements}

Authors are thankful to Professor Nitish K. Sanyal for the encouragement. One of the authors (R. S.) is thankful to C.S.I.R., New Delhi, for financial assistance.

17 D. W. Wood and N. W. Dalton, J. Phys. C 5, 1675 [1972].

18 D. L. Hunter and G. A. Baker, Jr., Phys. Rev. B 7, 3346 [1973].

19 H. E. Stanley, Introduction to Phase Transitions and Critical Phenomena, Clarendon, Oxford, England 1971, p. 90.

20 K. Pirnie, P. J. Wood, and J. Eve, Mol. Phys. 11, 551 [1966].

21 R. Shanker and R. A. Singh, Indian J. Pure and Appl. Phys. 12, 589 [1974].

22 R. E. Watson, M. Blume, and G. H. Vineyard, Phys. Rev. 181, 811 [1969].

23 C. Kittel, Introduction to Solid State Physics, John Wiley, New York 1966, p. 464.

24 J. P. Van Dyke and W. J. Camp, Phys. Rev. B 9, 3121 [1974].

25 W. J. Camp and J. P. Van Dyke (to be published).

26 D. S. Ritchie and M. E. Fisher, Phys. Rev. B 5, 2668 [1972].

27 L. J. de Jongh and A. R. Miedema, Adv. Physics 23, 1 [1974]. 